\title{
Vertex-Wise Shape Analysis of the Hippocampus: Disentangling Positional Differences from Volume Changes
}

\author{
Hosung Kim ${ }^{1}$, Tommaso Mansi ${ }^{2}$, Andrea Bernasconi ${ }^{1}$, and Neda Bernasconi ${ }^{1}$ \\ ${ }^{1}$ Department of Neurology and McConnell Brain Imaging Center, Montreal Neurological \\ Institute and Hospital, McGill University, Montreal, Quebec, Canada \\ ${ }^{2}$ Siemens Corporate Research, Image Analytics and Informatics, Princeton, NJ, USA \\ \{khs001, neda, andrea\}@bic.mni.mcgill.ca, \\ tommaso.mansiasiemens.com
}

\begin{abstract}
Hippocampal atrophy and developmental positional variants may cooccur in various neurological disorders. We propose a surface-based framework to analyze independently volume and positioning. After extracting the spherical harmonics combined with point distribution models (SPHARM-PDM) from manual labels, we computed displacement vectors between individual surfaces and the template. Then, we computed surface-based Jacobian determinants (SJD) from these vectors to localize volume changes. To analyze positional variants, we constructed a mean meridian axis (MEMAX), inheriting the shape-constrained point correspondences of SPHARM, on which we compute local curvatures and position vectors. We validated our method on synthetic shapes, and a large database of healthy subjects and patients with temporal lobe epilepsy. Our comprehensive analysis showed that in patients atrophy and positional changes cooccurred at the level of the posterior hippocampus. Indeed, in this region, while SPHARM-PDM showed mirrored deformations, SJD detected atrophy, and shape analysis of MEMAX unveiled medial positioning due to bending.
\end{abstract}

Keywords: surface, shape analysis, hippocampus, medial axis, Jacobian determinant, epilepsy.

\section{Introduction}

The most frequent drug-resistant epilepsy is temporal lobe epilepsy (TLE) related to hippocampal sclerosis, which generally appears as atrophy on MRI [1]. Detecting hippocampal atrophy is clinically relevant as patients have about $70 \%$ chance of becoming seizure free after surgery [2].

As atrophy reflects a single morphological characteristic, it may be insufficient to fully describe pathology. Indeed, in addition to atrophy, developmental anomalies, secondary to incomplete folding of the fetal hippocampus, may increase the susceptibility to TLE [3]. Such anomalies have been reported in other brain disorders in which the pathogenic mechanisms involve the temporal lobe, such as schizophrenia [4] and autism [5]. On MRI, hippocampal developmental anomalies appear as atypical 
shape and positioning of the hippocampus [3]. The complexity of the brain anatomy may hamper their visual identification, particularly when they co-occur with atrophy.

Advanced surface-based shape models, including deformation-based mapping and spherical harmonics description combined with point distribution models (SPHARMPDM), have allowed localization of morphological changes that may not be readily visible [6], and successfully identified hippocampal pathology in TLE [7] and schizophrenia [8]. The displacement metric provided by these approaches, however, does not allow differentiating volume changes from positional differences. Thus, a biologically meaningful interpretation of findings may be difficult, particularly when both morphological characteristics co-exist. Moreover, the concomitant presence of atrophy and positional changes, by increasing inter-subject shape variability, may reduce statistical sensitivity to detect subtle pathology.

A surface-based Jacobian metric has been proposed as a robust technique to assess volume changes [9]. Shape models based on the medial axis (i.e., the central path of a 3D object), on the other hand, allow quantifying local positional variations [8, 10]. Nevertheless, as these methods rely on coarse-scale sampling, they may lack sensitivity to assess high-dimensional features.

We propose a surface-based framework to analyze independently volume and positioning. We measured surface-based Jacobian determinants as in [9]. We construct a medial axis model that inherits the shape-constrained point correspondence of SPHARM-PDM, and quantifies fine-scale local positional vectors and curvature. We validated sensitivity and specificity using synthetic shapes, and assessed the pathological variations of the hippocampus by applying our method to a large database of healthy subjects $(n=46)$ and TLE patients $(n=78)$.

\section{Methods}

\subsection{Spherical Harmonic Description and Point Distribution Model (SPHARM- PDM)}

The hippocampal meshes are first extracted from manual labels and are parameterized using SPHARM-PDM, an area-preserving and distortion-minimizing spherical mapping that ensures point-wise correspondence through an icosahedron subdivision [6]. A template is then constructed by averaging all the individual SPHARM-PDM surfaces after rigid-body alignment. Individual surfaces are again rigidly aligned to this template to avoid any bias. Finally, vertex-wise displacement vectors are calculated between each individual and the template. The surface normal components of these vectors are used to quantify inward and outward deformations.

\subsection{Surface-Based Analysis of Volume Changes}

Volume changes are quantified on each vertex of the SPHARM-PDM surface as described in [9]. Briefly, we apply the heat equation to interpolate the vertex-wise displacement vectors within the volume domain enclosed by the surface boundary. The Jacobian determinant is then calculated from this interpolated vector field and projected back onto the SPHARM-PDM surface. Finally, after substracting 1 from the Jacobian determinant, growth $(J>0)$ or shrinkage $(J<0)$ of a unit-size cube is quantified along the surface-normal direction. 


\subsection{Construction of the Medial Axis}

Conventional approaches create the central path of a 3D object by joining points that are equidistant from the two closest facing points on the object's boundary [8, 10-11]. To provide inter-subject point correspondence, all individual skeletons are deformed into a common spatial frame by a thin plate registration [11]. Registration errors and smoothing in the resampling step may introduce biases in the subsequent analysis steps. In our approach, using the latitudes and longitudes parameters of SPHARMPDM, we parameterize the prime meridians of the object's two hemispheres (Fig 1A, B). Then we create a central route by performing pair-wise averaging of the prime meridians. This allows the route to inherit the shape-constrained correspondence of the SPHARM, thus minimizing biases, mainly those related to registration.

\subsubsection{Geometric Parameters of SPHARM}

The spherical harmonic basis functions $Y_{l}^{m}$ of degree $l$ and order $-l \leq m \leq l$ are defined by the spherical coordinates $\theta, \phi$ as:

$$
Y_{l}^{m}(\theta, \phi)=\sqrt{\frac{2 l+1(l-m) !}{4 \pi(l+m) !}} P_{m}^{l}(\cos \theta) e^{i m \phi} \quad Y_{l}^{-m}(\theta, \phi)=(-1)^{m} Y_{l}^{m^{*}}(\theta, \phi)
$$

In (1), $Y_{l}^{m^{*}}$ denotes the complex conjugate of $Y_{l}^{m}$ and $P_{l}^{m}$ the associated Legendre polynomials [6]. Summation of the spherical harmonics from 1 to $\mathrm{L}$ degree approximates a given structural boundary to a surface. A vertex point $\vec{s}$ on $\mathbf{S}$ is then uniquely determined by:

$$
\vec{s}(x, y, z)=\vec{s}(x(\theta, \phi), y(\theta, \phi), z(\theta, \phi))=\sum_{l=1}^{L} \sum_{m=-l}^{l} \vec{c}_{l}^{m} Y_{l}^{m}(\theta, \phi)
$$

The 3D weight vectors $\vec{c}_{l}^{m}$ are computed through least-square minimization. $\theta, \phi$ define latitudes and longitudes, respectively (yellow and green in Fig. 1A, B). Accordingly, $\vec{s}(0, \pi / 2)$ and $\vec{s}(\pi, \pi / 2)$ indicate the poles, and $\vec{s}(\pi / 2, \phi)$ the equator [6].

\subsubsection{Mean Meridian Axis (MEMAX)}

The functions $\vec{s}(\theta, 0)$ and $\vec{s}(\theta, \pi)$ in Eq. (2), describe a set of points that constitute the prime meridian (blue in Fig. 1A, B). The prime meridian points on a given hemisphere $\mathbf{s}=\vec{s}(0 \leq a \leq \pi / 2,0$ or $\pi)$ and their counterparts on its opposite side $\hat{\mathbf{s}}=\vec{s}(\pi / 2 \leq \pi-a \leq \pi, 0$ or $\pi)$ are averaged in a pair-wise fashion, thus yielding a mean meridian axis M, henceforth called MEMAX (red in Fig. 1C) as the skeleton of a shape.

For shape analysis, we sample $\mathrm{K}$ points on $\mathbf{M}$. We define $\mathbf{M}(\mathrm{k})$, where $\mathrm{k}=\{\mathrm{k} \mid 1$, $2, . ., \mathrm{K}\}$, using equidistant subdivisions of $\theta$, that is:

$$
\begin{aligned}
& \mathbf{M}(\mathrm{k})=\left\{\vec{s}\left(\frac{\pi}{2}-\frac{\pi}{2} \times \frac{2 k}{K}, 0\right)+\vec{s}\left(\frac{\pi}{2}+\frac{\pi}{2} \times \frac{2 k}{K}, 0\right)\right\} / 2, \quad \text { if } 1 \leq \mathrm{k} \leq \mathrm{K} / 2 \\
= & \left\{\vec{s}\left(\frac{\pi}{2} \times \frac{2(k-K / 2)}{K}, \pi\right)+\vec{s}\left(\frac{\pi}{2}+\frac{\pi}{2} \times \frac{2(k-K / 2)}{K}, \pi\right)\right\} / 2, \text { if } \mathrm{K} / 2 \leq \mathrm{n} \leq \mathrm{K}
\end{aligned}
$$




\subsection{Assessment of Local Positioning}

To assess shifting, group analysis is performed by comparing the location of each sample point on $\mathbf{M}$ using Hotelling $T^{2}$ metric [12] as:

$$
T^{2}=\left(\mu_{1}-\mu_{2}\right)^{\prime}\left(\sum_{1} \frac{1}{n_{1}}+\sum_{2} \frac{1}{n_{2}}\right)^{-1}\left(\mu_{1}-\mu_{2}\right)
$$

In this equation, $\mu_{1}, \mu_{2}$ are the group mean vectors, $\Sigma_{1}, \Sigma_{2}$ the group-separated covariance matrices and $\mathrm{n}_{1}, \mathrm{n}_{2}$ the number of samples in groups 1 and 2 .

The curvature at a given point $(\mathrm{x}, \mathrm{y}, \mathrm{z})$ of a $3 \mathrm{D}$ curve is generally computed using the Frenet-Serret formula. This measurement, however, provides only the degree of curving without distinguishing convexity from concavity, thus requiring additional visual inspection to fully understand its nature. Instead, we propose to measure the signed curvature varying along the normal directions of a surface defined by the prime meridian or the equator. We create an open surface $\mathbf{C}$ where its boundaries are defined by the prime meridian $\mathbf{s}(\mathrm{k})$ and $\hat{\mathbf{s}}(\mathrm{k})$. Since the MEMAX $\mathbf{M}(\mathrm{k})$ is a geometric mean of the two prime meridians, $\mathbf{M}(\mathrm{k})$ is placed in the middle $\mathbf{s}(\mathrm{k})$ and $\mathbf{s}(\mathrm{k})$ on the surface C (Fig. 1D left). We then calculate the Gaussian curvature $\kappa_{\text {Gauss }}$ at each sample point $\mathbf{M}(\mathrm{k})$ on $\mathbf{C}$ (Fig. 1D right). To differentiate the convexity (+) from the concavity (-), signs are computed using the normal vector of the $\mathrm{k}_{\mathrm{th}}$ facet on $\mathbf{C}$ $\mathbf{n}\left(\mathbf{C}_{\mathrm{k}}\right)=(\mathbf{M}(\mathrm{k}+1)-\mathbf{M}(\mathrm{k})) \times(\mathbf{s}(\mathrm{k})-\hat{\mathbf{s}}(\mathrm{k}))$, as:

$$
\operatorname{Sign}\left(\mathbf{n}\left(\mathbf{C}_{k}\right)\right)=\operatorname{Sign}\left(\mathbf{n}\left(\mathbf{C}_{k}\right) \bullet \mathbf{n}\left(\mathbf{C}_{k+1}\right) /\left\|\mathbf{n}\left(\mathbf{C}_{k}\right)\right\| \cdot\left\|\mathbf{n}\left(\mathbf{C}_{k+1}\right)\right\|\right)
$$

The signed curvature is finally determined as:

$$
\mathrm{K}=\operatorname{Sign}\left(\mathbf{n}\left(\mathbf{C}_{\mathrm{k}}\right)\right)\left|\kappa_{\text {Gauss }}\right|
$$

In the same fashion, we create the surface $\mathbf{C}^{\prime}$ on which boundaries are defined by the equator and place $\mathbf{M}(\mathrm{k})$ on $\mathbf{C}^{\prime}$. Then, we compute curvature $\mathrm{K}^{\prime}$ to measure changes along the normal to $\mathbf{C}^{\prime}$.

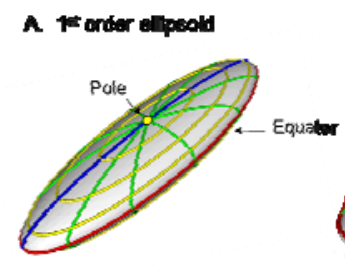

B. Hippocampal surface

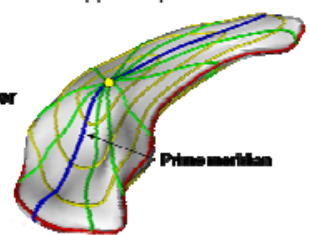

D.
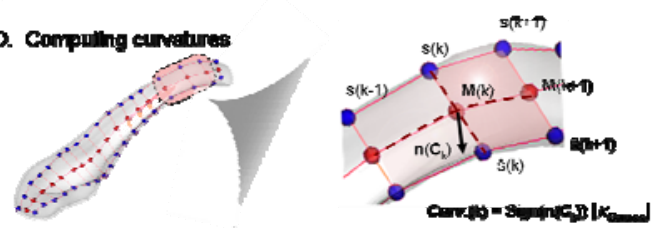

C. MEMAX

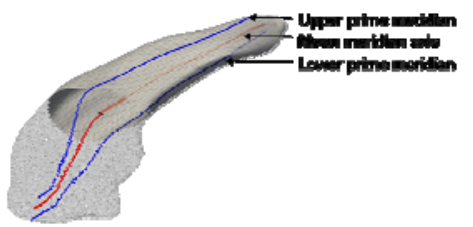

Fig. 1. Modeling of the mean meridian axis (MEMAX; A-C) and computing point-wise curvature (D). See text for details. 


\section{Experiments and Results}

\subsection{Synthetic Data}

We created a rounded cylindrical volume with a resolution of $0.25 \times 0.25 \times 0.25 \mathrm{~mm}^{3}$ and applied (Fig. 2A-C): $i$ ) bending, ii) local shifting (4.0 mm), and iii) simultaneous local shrinking $(0.5 \mathrm{~mm})$ and shifting $(4.0 \mathrm{~mm})$. We extracted the SPHARM-PDM surfaces and calculated the displacement vectors. Then, at each vertex, we computed the surface-normal component of the displacement vector (SNV) and the surfacebased Jacobian determinant (SJD) as a metric of local volume change. The MEMAX were extracted from all shapes. Finally, we quantified point-wise positioning of the variants compared to the reference.

The SNV showed mirrored inward/outward deformations on facing surfaces reflecting bending/shifting (Fig. 2D, top and middle). In case of simultaneous local shrinking and shifting, SNV did not enable differentiating shrinkage from shifting (Fig. 2D, bottom). Contrary to SNV, SJD was not influenced by bending/shifting (Fig. 2E, top and middle) and accurately localized the $0.5 \mathrm{~mm}$ local shrinkage (Fig. 2E, bottom). Analysis of MEMAX detected bending and local shifting only (Fig. 2F), and was robust to shrinking (Fig. 2F, middle and bottom).

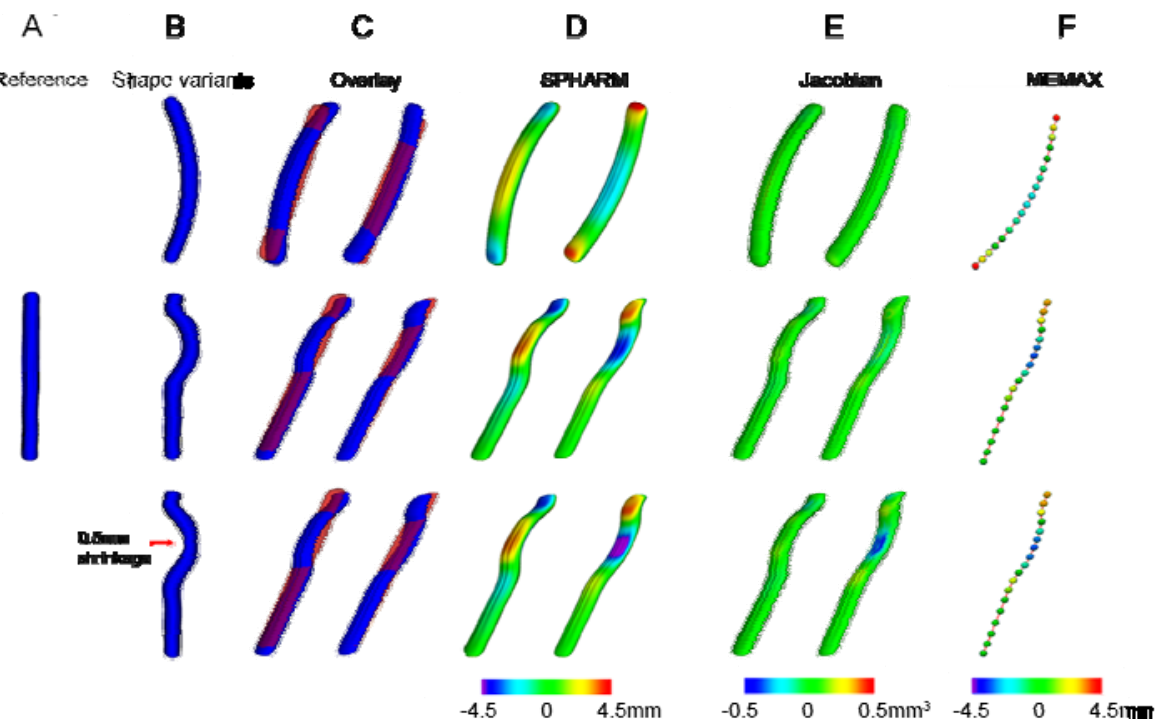

Fig. 2. Analysis of synthetic data. The $-/+$ signs indicate the direction of displacement (in D), volume (in $\mathbf{E}$ ), and shifting (in $\mathbf{F}$ ) with respect to the reference.

\subsection{Shape Analysis of the Hippocampus in Temporal Lobe Epilepsy}

\subsubsection{Subjects and Image Pre-processing}

We studied 78 drug-resistant TLE patients (age: $36 \pm 10$ ). Diagnosis and lateralization of the focus into left (LTLE; $n=34$ ) and right (RTLE; $n=44$ ) were determined by 
video-EEG and MRI evaluation. The control group consisted of 46 age- and sexmatched (age: $32 \pm 12$ ) healthy subjects. Our Ethics Committee approved this study.

High-resolution $1 \mathrm{~mm}$-isotropic $1.5 \mathrm{~T}$ MRIs were linearly registered to a stereotaxic space. An expert manually segmented the hippocampi in all subjects. Using z-score normalization based on healthy controls' distribution, all patients showed hippocampal atrophy (i.e. $\mathrm{z}<-2$ ) ipsilateral to the seizure focus.

\subsubsection{Optimization of Sampling Scale Along MEMAX}

We first extracted the MEMAX of the right hippocampi in controls and patients with RTLE. We then evaluated the effect of varying the total number of sample points (Ns = 2-36) on the sensitivity to detect positional changes (i.e., position vectors and Gaussian curvatures). For each individual and each metric, we averaged all sample points. We then performed group-wise Hotelling $\mathrm{T}^{2}$ test on the mean position vectors and Student's t-test on the mean curvatures. Results are shown in Fig. 3.

Comparing groups, we detected shifting in TLE with Ns ranging from 14 to 34 $\left(\mathrm{T}^{2}>3.6\right)$ and bending with Ns ranging from 14 to $22(\mathrm{t}>2.82)$. The mean inter-point distance spanned from 2.1 to $3.3 \mathrm{~mm}$. The sampling along MEMAX that provided the most significant group differences was $\mathrm{Ns}=18\left(\mathrm{~T}^{2}=3.71 ; \mathrm{t}=2.85\right)$. Thus, for the subsequent analyses, we used 18 points, with a mean inter-point distance of $2.7 \mathrm{~mm}$.
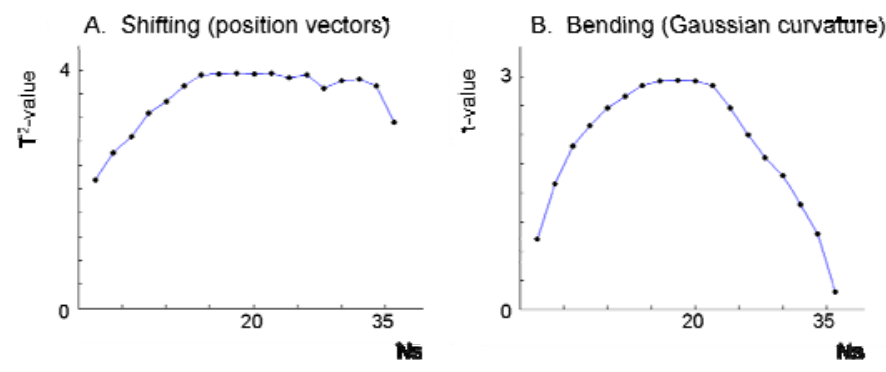

Fig. 3. Relationship between sampling points (Ns) along MEMAX and sensitivity in detecting shifting (A) and bending (B) in TLE patients

\subsubsection{Clinical Analysis of Local Hippocampal Shape Changes in TLE}

We performed vertex-wises group comparisons of surface-normal component of the displacement vector (SNV) and the surface-based Jacobian determinant (SJD), and point-wise comparisons of the Gaussian curvatures and positional vectors. We used the false discovery rate (FDR) to correct for multiple comparisons [13]. To localize changes, we schematically outlined hippocampal subfields on the surface template according to histological parcellations [7].

Results are presented in Fig. 4 A and B. Ipsilateral to the seizure focus, both SNV and SJD revealed areas of inward deformation and atrophy, respectively, in the hippocampal head and body (FDR $<0.001)$. In the tail, where SNV detected mirrored inward/outward deformations, SJD on the other hand unmasked atrophy. In addition, SJD detected small areas of CA1 inward deformations $(\mathrm{FDR}<0.05)$ in the contralateral hippocampus.

Curvature analysis of the mean meridian axis (MEMAX) detected ipsilateral bending towards the mid-sagittal plane (LTLE: 1 sample point, FDR=0.04; RTLE: 2 
sample points, FDR $<0.05$; Fig. 4D), at the transition between hippocampal body and tail. We also found ipsilateral medial shifting at the level of the tail (LTLE: 3 points, FDR $<0.01$; RTLE: 6 points, FDR $<0.05$; Fig. 4E), which overlapped with the region of mirrored inward/outward deformations detected by SNV (Fig. 4A).

\section{A. SPHARMPDM}

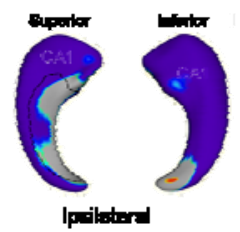

Inward

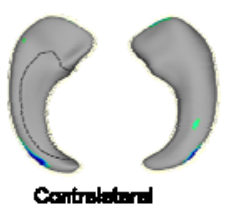

Outward

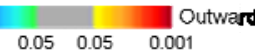

c.

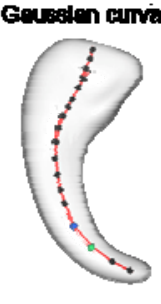

Ipediateral

D. Postition vactor

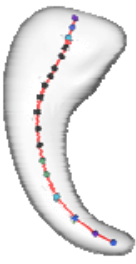

Ipetlatherel
B. 8urteco-besed Jecoblan

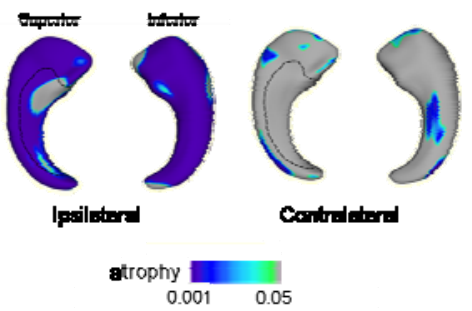

E. Woen of MEmax

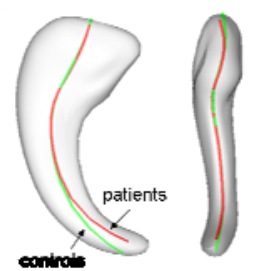

Fig. 4. Comparisons between TLE and controls for SPHARM-PDM (A), Surface-based Jacobian $(\mathbf{B})$, Gaussian curvature $(\mathbf{C})$, position vectors $(\mathbf{D})$. The geometric means of MEMAX $(\mathbf{E})$ is shown to ease the interpretation of C/ D. Color-scales show FDR-corrected p-values.

\section{Discussion and Conclusion}

We previously proposed [9] to quantify local volume changes by computing SJD. In case of co-occurrence of volume and positional anomalies this method failed to quantify the latter. Here we unambiguously disentangled volume from positional changes by quantifying independently both morphological characteristics, i.e. by calculating SJD and Gaussian curvatures on the mean meridian axis (MEMAX).

In agreement with histology [14], SJD detected atrophy in TLE encompassing the CA1 subfield. Moreover, our analysis showed that atrophy and positional changes cooccurred in the hippocampal tail. In this region, while SPHARM-PDM showed mirrored deformations, SJD detected atrophy, and shape analysis of MEMAX unveiled medial positioning due to bending.

In conventional medial models, a small number of sample points (e.g., 8) are used mainly to reduce registration errors $[8,10-11]$. This relatively coarse sampling may reduce sensitivity to subtle changes. By using geometric parameters of SPHARM [6], MEMAX allowed shape-constrained correspondence without a registration step. We found that modulating the number of sample points influenced the sensitivity to detect positional changes, with the largest changes at a finer scale of 18 points. 
A single longitudinal central path would not allow measuring thickness nor retrieve the original shapes of an object. Our model was specifically designed to quantify positional changes. To achieve this, conventional medial representations would require additional processing steps, such as pruning [10-11], to eliminate undesirable secondary branches producing irregular skeleton topologies.

Medial positioning is a feature of hippocampal developmental abnormalities [3] that may predispose to TLE. Disentangling atrophy from positioning anomalies may provide new insights in the pathogenesis of a variety of other brain disorders, such as schizophrenia and autism, in which these morphometric characteristics coexist.

\section{References}

1. Berkovic, S.F., Andermann, F., Olivier, A., Ethier, R., Melanson, D., Robitaille, Y., Kuzniecky, R., Peters, T., Feindel, W.: Hippocampal sclerosis in temporal lobe epilepsy demonstrated by magnetic resonance imaging. Ann. Neurol. 29(2), 175-182 (1991)

2. Schramm, J., Clusmann, H.: The surgery of epilepsy. Neurosurgery 62(suppl. 2), 463-481 (2008); discussion 481

3. Bernasconi, N., Kinay, D., Andermann, F., Antel, S., Bernasconi, A.: Analysis of shape and positioning of the hippocampal formation: An mri study in patients with partial epilepsy and healthy controls. Brain 128(Pt. 10), 2442-2452 (2005)

4. Connor, S.J., Ng, V., McDonald, C., Schulze, K., Morgan, K., Dazzan, P., Murray, R.M.: A study of hippocampal shape anomaly in schizophrenia and in families multiply affected by schizophrenia or bipolar disorder. Neuroradiology 46(7), 523-534 (2004)

5. Salmond, C.H., Ashburner, J., Connelly, A., Friston, K.J., Gadian, D.G., Vargha-Khadem, F.: The role of the medial temporal lobe in autistic spectrum disorders. European Journal of Neuroscience 22(3), 764-772 (2005)

6. Styner, M., Oguz, I., Xu, S., Brechbuhler, C., Pantazis, D., Gerig, G.: Statistical shape analysis of brain structures using spharm-pdm. In: MICCAI 2006 Opensource Workshop (2006)

7. Hogan, R.E., Wang, L., Bertrand, M.E., Willmore, L.J., Bucholz, R.D., Nassif, A.S., Csernansky, J.G.: MRI-based high-dimensional hippocampal mapping in mesial temporal lobe epilepsy. Brain 127(8), 1731-1740 (2004)

8. Styner, M., Pantazis, D., Gerig, G.: Boundary and medial shape analysis of the hippocampus in schizophrenia. Med. Image Anal. 8(3), 197-203 (2004)

9. Kim, H., Besson, P., Colliot, O., Bernasconi, A., Bernasconi, N.: Surface-based vector analysis using heat equation interpolation: A new approach to quantify local hippocampal volume changes. In: Metaxas, D., Axel, L., Fichtinger, G., Székely, G. (eds.) MICCAI 2008, Part I. LNCS, vol. 5241, pp. 1008-1015. Springer, Heidelberg (2008)

10. Joshi, S., Pizer, S., Fletcher, P.T., Yushkevich, P., Thall, A., Marron, J.S.: Multiscale deformable model segmentation and statistical shape analysis using medial descriptions. IEEE Trans. Med. Imaging 21(5), 538-550 (2002)

11. Styner, M., Gerig, G., Lieberman, J., Jones, D., Weinberger, D.: Statistical shape analysis of neuroanatomical structures based on medial models. Medical Image Analysis 7(3), 207-220 (2003)

12. Seber, G.F.: Multivariate observations, vol. 686. John Wiley \& Sons Inc., Chichester (1984)

13. Benjamini, Y., Hochberg, Y.: Controlling the false discovery rate. J. Royal Stat. Soc. 57(1), 289-300 (1995)

14. Blumcke, I., Thom, M., Wiestler, O.D.: Ammon's horn sclerosis: A maldevelopmental disorder associated with temporal lobe epilepsy. Brain Pathol. 12(2), 199-211 (2002) 